microcrystallography at BL41XU and the future plan of the real micro beam beamline.

\section{MS36 P07}

Application of a pn-CCD as 2D energy dispersive detector Wolfram Leitenberger ${ }^{\mathrm{a}}$, Robert Hartmann, ${ }^{\text {b,c }}$ Ullrich Pietsch, ${ }^{\mathrm{d}}$ Lothar Strüder ${ }^{\mathrm{e}, \mathrm{c}}{ }^{\mathrm{a}}$ Universität Potsdam, Institut für Physik, Germany. ${ }^{\mathrm{b}} M P I$ Halbleiterlabor, München Germany, ${ }^{c} P N$ Sensor GmbH München, Germany, ${ }^{d}$ Institut. f. Physik Universität Siegen, Germany, ${ }^{e}$ MPI f. extraterrestrische Physik Garching, Germany .Email: leitenberger@uni-potsdam.de

Keywords: x-ray diffraction, energy dispersive detector, spectroscopy

We present our first results with a pn-CCD detector used for X-ray diffraction experiments with white synchrotron radiation. The pn-CCD was developed for spectroscopy and imaging in a wide spectral range from 0.2 to $30 \mathrm{keV}$ $\mathrm{X}$-rays for astronomy applications [1]. It consists of an image area of $19 \times 19 \mathrm{~mm}^{2}(256 \times 256$ pixel $)$ and a frame store area of the same pixel number. The readout was done by 256 parallel readout channels in approximately $5 \mathrm{~ms}$ (200 frames per second). At low photon intensity every pixel can be used as an energy dispersive detector. Finally one obtains the position and the energy of each incoming photon which allows to perform new interesting types of experiments.

We performed test experiments like X-ray powder diffraction and $\mathrm{X}$-ray reflectivity studies at organic thin films both using an incoming white X-ray beam. Here characterized the device in terms of energy resolution and spatial resolution and tested several readout modes of the freely programmable readout system. The measured data are discussed and plans for future experiments are presented.

[1] Strüder, L. et al., Astronomy \& Astrophysics, 2001, 365, 1826.

\section{MS36 P08}

New high-temperature sample stage for combined reflection- and transmission-PXRD $\mathrm{C} \quad \mathrm{Resch}^{\mathrm{a}}$, C.Weidenthaler ${ }^{\mathrm{b}}$, P.Hofbauer ${ }^{\mathrm{a}}$, P.Schwarzl ${ }^{\mathrm{a}}$, ${ }^{\mathrm{a}}$ Anton Paar GmbH, Graz, Austria. b Max-Planck-Institut für Kohlenforschung, Mülheim a.d.R., Germany. E-mail: christian.resch@anton-paar.com

Keywords: X-ray diffractometer instrumentation, capillary heater, high-temperature sample stage

It is often necessary in powder XRD to measure samples in capillaries because the sample material is either not stable in air, available in very small amounts only or very X-ray transparent. A new add-on for the Anton Paar HTK1200N high-temperature sample stage has been developed, which turns the HTK1200N into a capillary heater up to $1200{ }^{\circ} \mathrm{C}$. The add-on consists of a horizontal capillary spinner that is mounted on the front plate of the sample stage. The capillary is heated very uniformly with a surrounding heater, the temperature is accurately measured with a thermocouple close to the capillary. Standard capillaries can be used, and it is possible to mount open and sealed capillaries. The poster presents the design of the new instrument and its technical data. It shows application examples for the investigation of $\mathrm{Cu} / \mathrm{Zn} / \mathrm{Al}$ catalysts and mesoporous materials. 